\title{
Diffusion of a mono-energetic implanted species with a Gaussian profile
}

Johan B. Malherbe*, P.A. Selyshchev, O.S. Odutemowo, C.C. Theron, E.G. Njoroge, D.F. Langa, T.T. Hlatshwayo

Department of Physics, University of Pretoria, Pretoria, 0028, South Africa

\section{Highlights}

- General solution to the Fick diffusion equation with an initially Gaussian profile.

- Analyses the dominating property of this solution.

- Compares approximate models/solutions to the exact solution.

\begin{abstract}
The implanted profile in an isotropic substrate of a mono-energetic ion species is usually very near a Gaussian profile. An exact solution to the time-dependent Fick diffusion equation of an initially Gaussian profile is presented. This solution is a general one also covering the diffusion within the two limiting cases usually considered in solutions to the Fick equation, viz. a perfect sink at the surface and a perfectly reflecting surface plane at the surface. An analysis of the solutions for these two cases shows that at small diffusion times the main effect of annealing is a nearly symmetric broadening of the implanted profile. At the origin and for longer diffusion times the profile deviates significantly from Gaussian. A review is also given of past attempts to extract diffusion coefficients by fitting experimental data to approximate equations based on simplified initial profiles.
\end{abstract}

Key words: Fick diffusion equation, Gaussian profile, implanted profile, diffusion, doping,

* Corresponding author. johan.malherbe@up.ac.za 


\section{INTRODUCTION}

There are several applications for ion implantation into solid substrates. The most common application is in the semiconductor industry for doping semiconductors [1] Despite its higher cost, the main advantage that doping via ion implantation has over the in-diffusion process is better control over the doping level and uniformity of the dopant over the surface of the wafer. These result in a significant improvement in the quality of the ICs. To electrically activate the implanted dopants (i.e. to allow them to move into substitutional sites in the substrate crystal structure) and to repair the radiation damage, the substrate must be annealed, either thermally (typically about $1000{ }^{\circ} \mathrm{C}$ for 30 minutes) or by rapid thermal annealing.

Although the applications of ion implantations are traditionally mainly limited to the semiconductor industry, there are also some applications where ion implantations and subsequent annealing are being investigated and employed in insulators - see Ref. [2] for some examples. There are also limited applications of nitrogen and other ions implantations in metals (e.g. drill bits) and in some medical applications (e.g. prosthetic devices such as artificial joints) for the purpose of hardening, increasing wear resistance and preventing crack propagation [3, 4]. An additional benefit of this implantation is often also an increase in corrosion resistance of the substrate.

In all of these applications it is usually important to know the extent by which the implants will diffuse by thermal annealing. In the semiconductor applications diffusion of the dopants is, for obvious reason, usually detrimental. However, for many of the other applications, e.g. hard, anti-corrosive layers on metals, diffusion is an advantage because it increases the depth of the layer. Consequently, there are numerous studies investigating the diffusion of implanted elements in metals $[5,6]$, semiconductors like $\mathrm{Si}[7,8]$ and $\mathrm{SiC}[9,10]$, and insulators $[11,12]$.

Due to the statistical nature of the collisions between energetic impinging ions with the substrate atoms, the implanted profile is usually very near a Gaussian. Consequently, for most calculations and most practical examples it is usually assumed that the implanted profile is purely Gaussian - an assumption also made in this paper. 
It is only in exceptional cases (e.g. channeling) that there is a large deviation from a Gaussian profile.

In this paper, an exact general solution to the time-dependent Fick diffusion equation is given for an originally Gaussian implanted profile is given. A review is also given of past attempts to extract diffusion coefficients by fitting experimental data to approximate equations based on simplified initial profiles. It is shown that some of these can result in diffusivity values which are very near the real ones.

\section{THEORY}

The time-dependent Fick diffusion differential equation $[13,14]$ is given by

$$
\frac{\partial N}{\partial t}=D \frac{\partial^{2} N}{\partial x^{2}}
$$

where $D$ is the diffusion coefficient (usually taken as a constant) and $N(x, t)$ is the number of diffusing particles per unit volume at time $t$ and distance $x$. In order to obtain a general and correct solution for a pure Gaussian implanted profile, we follow Boltaks [14] which gives a general solution in the limit of low impurity concentration, for an isotropic, non-time dependent, semi-infinite body (i.e. $0<\mathrm{x}<\infty$ ) and $t>0$ to the Fick diffusion differential equation

$$
N(x, t)=\frac{1}{2 \sqrt{\pi D t}} \int_{o}^{\infty}\left[N(\xi, 0) \exp \left(-\frac{(\xi-x)^{2}}{4 D t}\right)+N_{I}(-\xi, 0) \exp \left(-\frac{(\xi+x)^{2}}{4 D t}\right)\right] d \xi
$$

where $N(\xi, 0)$ is the original distribution of the impurity and $N_{I}(\xi, 0)$ is a function that must be determined from the boundary conditions. Suppose the number of implanted particles per unit volume at $x=0$ for $\mathrm{t}>0$ is a constant $N_{0}$, i.e.

$$
N(0, t)=\frac{1}{2 \sqrt{\pi D t}} \int_{0}^{\infty}\left[N(\xi, 0)+N_{I}(-\xi, 0)\right] \exp \left(-\frac{(\xi)^{2}}{4 D t}\right) d \xi=N_{0}
$$


An elementary relationship which still satisfies the other boundary condition for the above solution to (1), viz. $N(x, t) \rightarrow 0$ as $x \rightarrow \infty$, is

$$
N_{I}(-\xi, 0)=-k N(\xi, 0)
$$

with $k$ a constant and

$$
N(x, t)=\frac{1}{\sqrt{\pi D t}} \int_{0}^{\infty} N(\xi, 0)\left[\exp \left(-\frac{(\xi-x)^{2}}{4 D t}\right)-k \exp \left(-\frac{(\xi+x)^{2}}{4 D t}\right)\right] d \xi
$$

Note that the two extreme cases for $k$ in (5) are general solutions given in Boltaks [14]. $k=-1$ represents the case of a perfectly reflecting boundary at $x=0$ with zero flux at the origin, i.e. $\left.j(x, t)\right|_{x=0}=-\left.D \frac{\partial}{\partial x} N(x, t)\right|_{x=0}=0$ and therefore no loss of impurity material from the substrate); while $k=1$ represents in the case of a perfect sink at the surface of the substrate meaning that any impurity arriving at the surface will immediately sublimate [14]. The general solution with $-1<k<1$, will lead to a time dependent surface concentration

$$
N(0, t)=C_{0}(t)
$$

Equation (4) determines the function $N_{I}(-\xi, 0)$. For an isotropic medium implanted with mono-energetic impurity atoms, the initial profile is assumed to be purely Gaussian with projected range $R_{p}$ and range straggling $\Delta R_{p}$, i.e.

$$
N(\xi, 0)=A_{0} \exp \left(-\frac{\left(\xi-R_{p}\right)^{2}}{2 \Delta R_{p}^{2}}\right)
$$

Substituting (7) for $N(\xi, 0)$ in (5) and calculating the integrals by completing the squares in the arguments of the exponential functions and then using the identity [15] for $a \neq 0$ 


$$
\int \exp \left[-\left(a x^{2}+2 b x+c\right)\right] d x=\frac{1}{2} \sqrt{\frac{\pi}{a}} \exp \left(\frac{b^{2}-a c}{a}\right) \operatorname{erf}\left(\sqrt{a} x+\frac{b}{\sqrt{a}}\right)+\text { constant }
$$

one eventually obtains the solution

$$
\begin{aligned}
& N(x, t)= \\
& \frac{A_{0} \cdot \Delta R_{p}}{2 \sqrt{2 D t+\Delta R_{p}^{2}}} e^{\left(-\frac{\left(x-R_{p}\right)^{2}}{4 D t+2 \Delta R_{p}^{2}}\right)}
\end{aligned}\left[\begin{array}{c}
1+\operatorname{erf}\left(\frac{2 D t R_{p}+x \Delta R_{p}^{2}}{\Delta R_{p} \sqrt{2(2 D t)^{2}+4 D t \Delta R_{p}^{2}}}\right)- \\
\left.k e^{\left(-\frac{x R_{p}}{D t+\Delta R_{p}^{2} / 2}\right)}\left\{1+\operatorname{erf}\left(\frac{2 D t R_{p}-x \Delta R_{p}^{2}}{\Delta R_{p} \sqrt{2(2 D t)^{2}+4 D t \Delta R_{p}^{2}}}\right)\right\}\right]
\end{array}\right.
$$

and

$$
k=1-\left[\frac{2 C_{0}}{A_{0}} \frac{\sqrt{2 D t+\Delta R_{p}^{2}}}{\cdot \Delta R_{p}} \exp \left(\frac{R_{p}^{2}}{4 D t+2 \Delta R_{p}^{2}}\right) \quad\left\{1+\operatorname{erf}\left(\frac{R_{p} \sqrt{D t}}{\Delta R_{p} \sqrt{2 D t+\Delta R_{p}^{2}}}\right)\right\}\right]
$$

The solution (9) was also derived by Strohm [16] but only for the much simpler limiting cases where either $k=1$ or $k=-1$, i.e. that of a perfect sink at the surface and of a perfect reflecting surface. The general solution (9) together with (10) represents the more usual case for shallow implants where some of the impurity reaching the surface is released into the environment (i.e. loss of the diffusant/impurity) leaving a non-zero surface concentration of the impurity, given by

$$
N(0, t)=\frac{(1-k) K}{4 \sqrt{\pi D\left(t+t_{0}\right)}} e^{\left(-\frac{\left(R_{p}\right)^{2}}{4 D\left(t+t_{0}\right)}\right)}\left\{1+\operatorname{erf}\left(\frac{t R_{p}}{\sqrt{4 D t t_{0}\left(t+t_{0}\right)}}\right)\right\}=C_{0}(t)
$$


Although, equation (4) determines the function $N_{I}(-\xi, 0)$, equation (11) is the more practical parameter to use when fitting experimental data to the general solution (9 $10)$.

In the following simulated diffusion profiles are considered for the two limiting cases, $k=1$ and $k=-1$. For all the model simulations typical implantation parameter values were used for the initial Gaussian profile, viz. $R_{p}=120 \mathrm{~nm}, \Delta R_{p}=30 \mathrm{~nm}, A_{0}=0.66$ atomic percent, and also a realistic diffusion coefficient $D=1 \times 10^{-19} \mathrm{~m}^{2} \mathrm{~s}^{-1}=0.1 \mathrm{~nm}^{2}$ $\mathrm{s}^{-1}$. The implantation parameters were chosen to depict a deep implanted profile where the surface concentration of the implanted material is effectively zero. Furthermore, the concentration of the implanted impurity is low to comply with Fickian diffusion conditions.

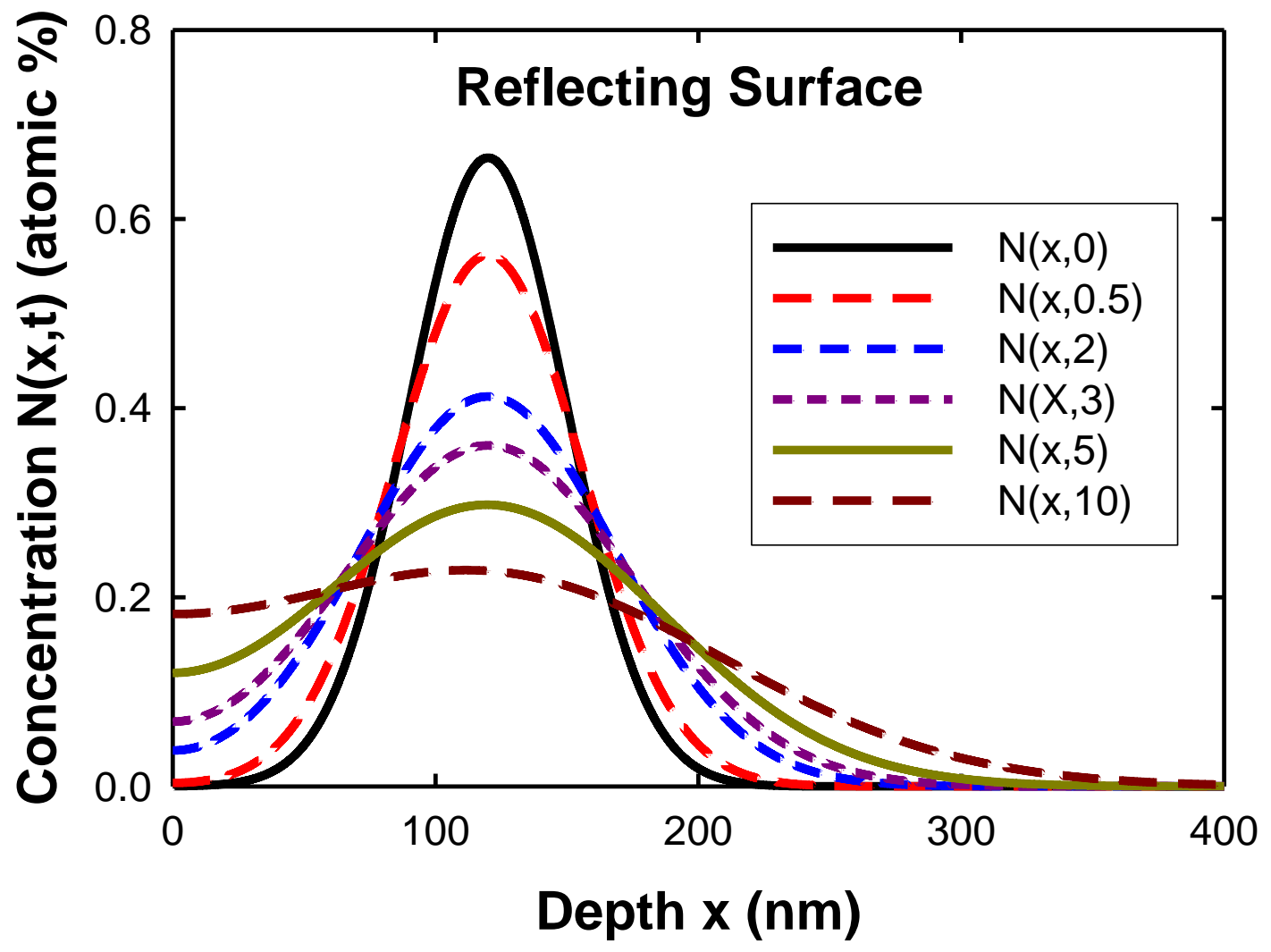

Figure 1. A simulated diffusion profile, i. e. equations (9) and (10), for the case $k=-1$ (i.e. a perfectly reflecting boundary at $x=0$ ), is given for different diffusion times $t$ (in hours) indicated in the figure. Typical implantation values were used for initial Gaussian profile: $R_{p}=120 \mathrm{~nm}, \Delta R_{p}=30 \mathrm{~nm}, A_{0}=$ 0.66 atomic percent, and a diffusion coefficient $D=0.1 \mathrm{~nm}^{2} \mathrm{~s}^{-1}$. 
In Figure 1 a simulated diffusion profile, for the case $k=-1$, is given at different times $t$. At the two lowest diffusion times the diffused profile is still fairly Gaussian. The main visible effect of the diffusion is the fairly symmetric broadening of the original Gaussian profile and a lowering of the maximum peak height due to this broadening of the peak. The (depth) position of maximum peak height has not really shifted away from the $R_{p}$ value of the original Gaussian profile. For larger diffusion times, the peak becomes increasingly asymmetric with the maximum peak height position shifting towards the surface to eventually coincides with $x=0$, i.e. the surface of the substrate. These two effects occur because the implanted (impurity) atoms that diffuse towards and reach the surface are reflected back with no material being lost from the substrate. Due to the diffusion process being fundamentally a random walk process, there is conglomeration at the surface. For increasingly long diffusion times the impurity atoms diffuse deeper inside the surface with an increasing lowering of the peak concentration at the surface.

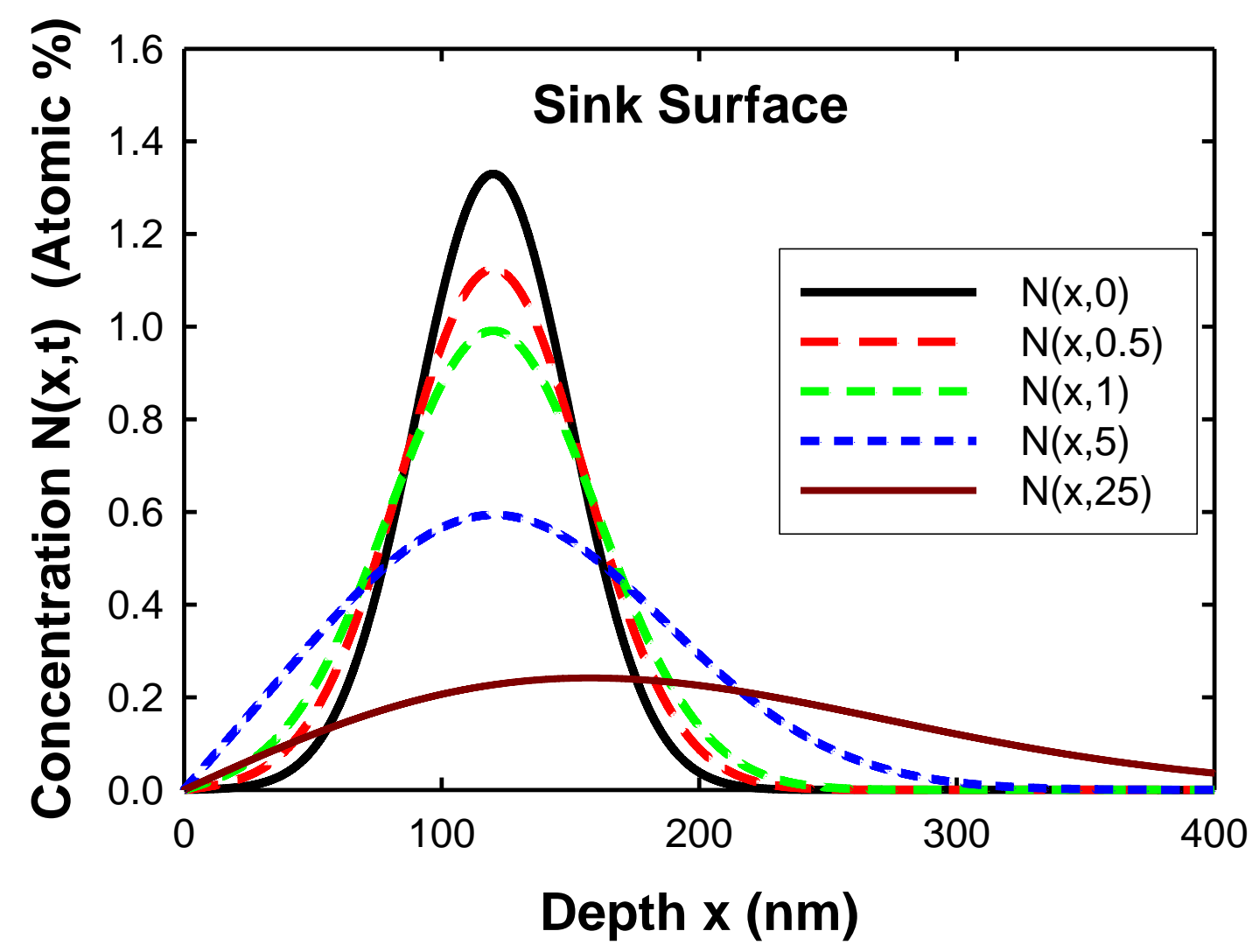

Figure 2. A simulated diffusion profile, i. e. equations (9) and (10), for the case $k=1$ (i.e. a perfect sink at the surface), is given for different diffusion times $t$ (in hours) indicated in the figure. Typical implantation values were used for initial Gaussian profile: $R_{p}=120 \mathrm{~nm}, \Delta R_{p}=30 \mathrm{~nm}, A_{0}=0.66$ atomic percent, and a diffusion coefficient $D=0.1 \mathrm{~nm}^{2} \mathrm{~s}^{-1}$. 
In Figure 2 the same parameters used in Figure 1 are used in a simulated diffusion profile, for the case $k=1$. This case represents a perfect sink for the impurity at the surface. In practical terms it illustrates the diffusion of an impurity with a high vapour pressure at the annealing times leading to a complete sublimation of the impurity atoms into the environment when they reach the surface of the substrate. This is a more common occurrence than the previous example considered in Figure 1. The same observations as seen in Figure 1, can be made here for the lower diffusion times, i.e. a symmetric broadening of the peak, lowering of the peak height and nearly no change in the maximum peak height position. For values near $x=0$, the boundary condition of a sink, forces the diffused profile to become 0 and the diffused profiles deviate from Gaussian, especially for larger diffusion times. For the three lowest times there was insignificant loss of impurity material. At the largest diffusion time, i.e. $\mathrm{t}=25 \mathrm{~h}$, there is clearly a loss of impurity material (about $40 \%$ ) with the profile now obviously no longer Gaussian but significantly skewed. The position of maximum peak height has also shifted away from the $R_{p}$ value to deeper values inside the substrate. These tendencies are enhanced (not shown) at even longer diffusion times (i.e. larger $D t$ values).

\section{DISCUSSION}

That both diffused profiles for the two limiting cases for the parameter $k$ in (10) remain largely Gaussian in shape at the lower diffusion times, is due to the fact that the error function $\operatorname{erf} z \approx 1$ for $\mathrm{x} \gg>1$. Thus, the pre-(square) bracket term in (9), i.e.

$$
\frac{A_{0} \cdot \Delta R_{p}}{\sqrt{2 D t+\Delta R_{p}^{2}}} e^{\left(-\frac{\left(x-R_{p}\right)^{2}}{4 D t+2 \Delta R_{p}^{2}}\right)}
$$

is the dominating term in (9) except for small $x$ and large $t$ values. This is a Gaussian function, which is broader than the original implanted distribution, due to factor $4 D t$ in the quotient of the argument in the exponential function. Consequently, it is convenient to fit the experimental values first to such a Gaussian function to obtain an approximate solution for the diffusion coefficient $D$, which then together with the estimates for $N_{0}$ and $A_{0}$, result in an efficient fitting of the full equation (9-10). 
Note that the notation $2 D t=\Delta R_{D}{ }^{2}$ and $\Delta R_{p}{ }^{2}=2 D t_{0}$ makes it clear that the term

$$
4 D t+4 D t_{0}=2 \Delta R_{D}^{2}+2 \Delta R_{p}^{2}=2 \sigma^{2}
$$

represents the second moment of the Gaussian fit, with $2 D t=\Delta R_{D}{ }^{2}$ representing the increased broadening of the profile due to diffusion.

The above solution(s) only apply for Fickian diffusion, i.e. a random walk diffusion process. When there is an addition force mechanism influencing causing a preferential direction for the diffusion atoms, such as Gibbsian segregation, then the solution is no longer valid.

The energetic bombarding ions can cause several effects in the substrate material which can influence the diffusion kinetics and mechanisms. The most obvious of these effects is radiation damage which can lead to radiation enhanced and/or radiation induced diffusion [17]. The above solution(s) can handle these cases as long as the diffusion is still Fickian diffusion. Radiation damage can also result in the trapping of the implanted species by defect complexes [18]. These diffusion traps are temperature dependent, usually causing no or little diffusion below the trap releasing temperature, and then normal diffusion above that temperature. The above solution(s) can then be applied to determine the diffusion coefficients above at these higher temperatures. Another phenomenon which also acts as a kind of diffusion trap is the chemical compound formation between the implanted atoms and substrate atoms. Sputtering of and substrate topography development on the substrate, due to the ion bombardment, do not affect the diffusion mechanisms but can lead to erroneous depth profiles $[17,19]$.

An example of the diffusion of $200 \mathrm{keV}$ implanted $\mathrm{Sr}^{+}$ions into a glassy carbon substrate to a fluence of $2 \times 10^{16} \mathrm{Sr}^{+} \mathrm{cm}^{-2}$ is shown in Figure 3 to illustrate the application of the above solution(s). Since glassy carbon is basically a semiamorphous material, the implanted strontium profile is very near a Gaussian profile [20]. The implanted glassy carbon was heated for $1 \mathrm{~h}$ in vacuum. Both the asimplanted $\mathrm{Sr}$ profile and the diffused profiles were determined by Rutherford 
backscattering (RBS). Since the Sr signals at the surface $(x=0)$ for both profiles were zero, the diffused profile was fitted to the solution for a sink at the surface. In fitting the $\mathrm{Sr}$ profile, the projected range $R_{P}$ was also used as a fitting parameter because dynamic annealing resulted in a shift of the whole profile towards the bulk of the glassy carbon. The fitting gave a diffusion coefficient of $D=5.7 \times 10^{-19} \mathrm{~m}^{2} \mathrm{~s}^{-1}$.

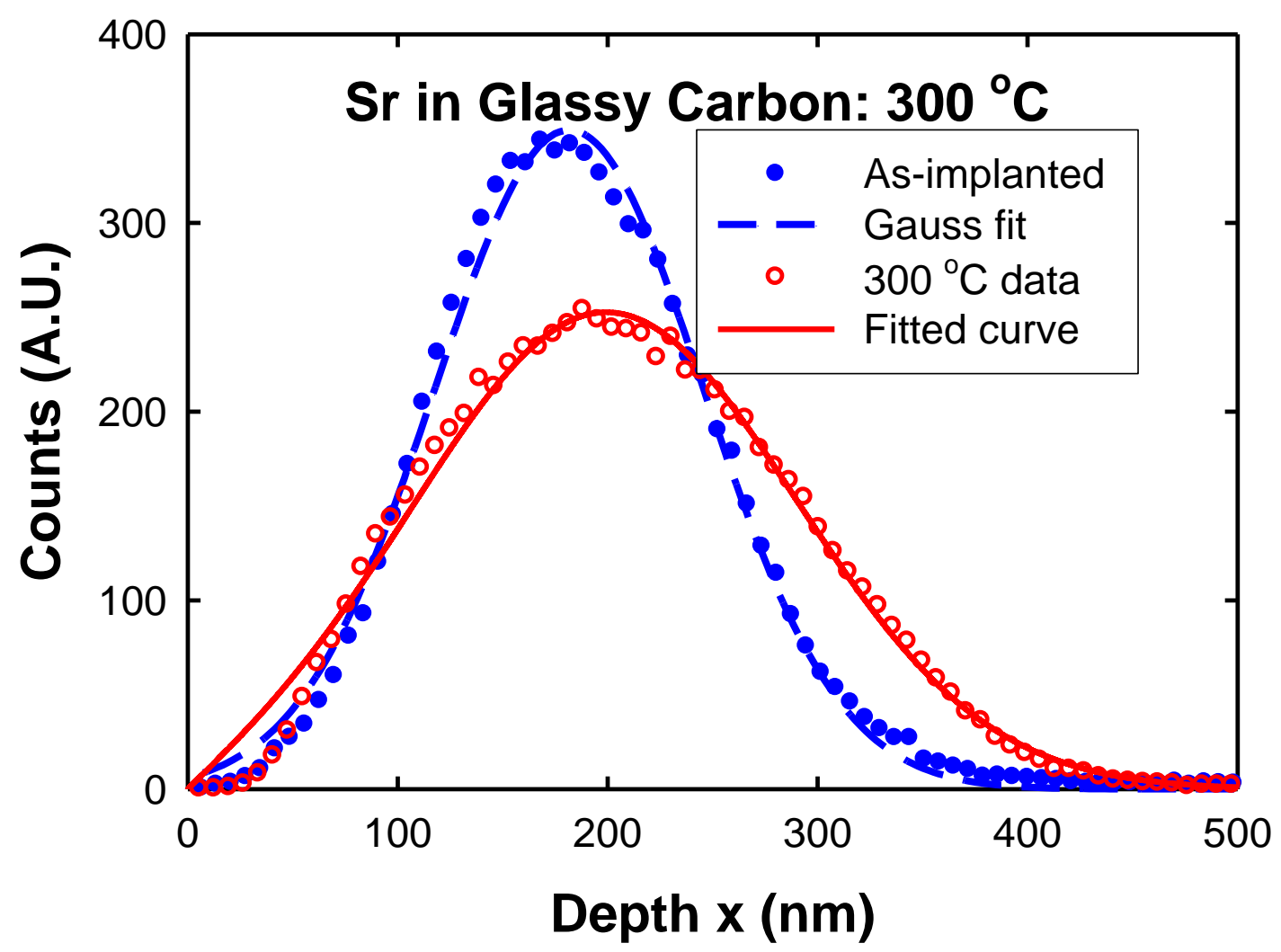

Figure 3. Diffusion of $200 \mathrm{keV}$ implanted strontium ions into glassy carbon aand annealed at $300{ }^{\circ} \mathrm{C}$ for $1 \mathrm{~h}$. The as-implanted Sr RBS measurements (full blue circles) were fitted to a Gaussian function (blue curve) while the diffused profile (open red circles) were fitted to (9) and (10) for $k=1$, i.e. the sink solution (red curve).

\section{COMPARISONS WITH OTHER EQUATIONS}

There had been a few approaches in the past for analysing the diffusion of a Gaussian implanted species in a substrate. In the one approach, simplified initial conditions are chosen where the impurity profile is assumed to have a constant concentration. Typically, a finite or infinite layer is assumed for the impurity profile - see for 
example $[6,21,22]$. We shall consider both these two cases below for parameters relevant to the implanted profile (6).

For an finite embedded layer of width $2 \Delta R_{p}$ centred around $R_{p}$, i.e. the region $\left(R_{p}-\right.$ $\left.\Delta R_{p}, R_{p}+\Delta R_{p}\right)$ where $N(x, 0)=N_{0}$ in an infinite substrate, the following error function solution is obtained $[13,14]$

$$
N_{E}(x, t)=\frac{N_{0}}{2}\left[\operatorname{erf}\left(\frac{\Delta R_{p}-R_{p}+x}{2 \sqrt{D t}}\right)+\operatorname{erf}\left(\frac{\Delta R_{p}+R_{p}-x}{2 \sqrt{D t}}\right)\right]
$$

In (14) we added the subscript $E$ (for embedded layer) to distinguish it from our solution $N$ in (8). Note that $N_{0}$ must be within the solubility limit of the impurity in the medium. In Figure 4 the original embedded layer profile and the equivalent original implanted profile are given. Both these two profiles represent the same number of impurity atoms, i.e. the areas under the two curves are the same. For the exact diffusion equation we assumed a perfect sink at the surface, i.e. $k=1$. Both diffused profiles (i.e. (9) and (14)) are also shown for a constant diffusivity $D(=10)$ and different time $t$ values. 


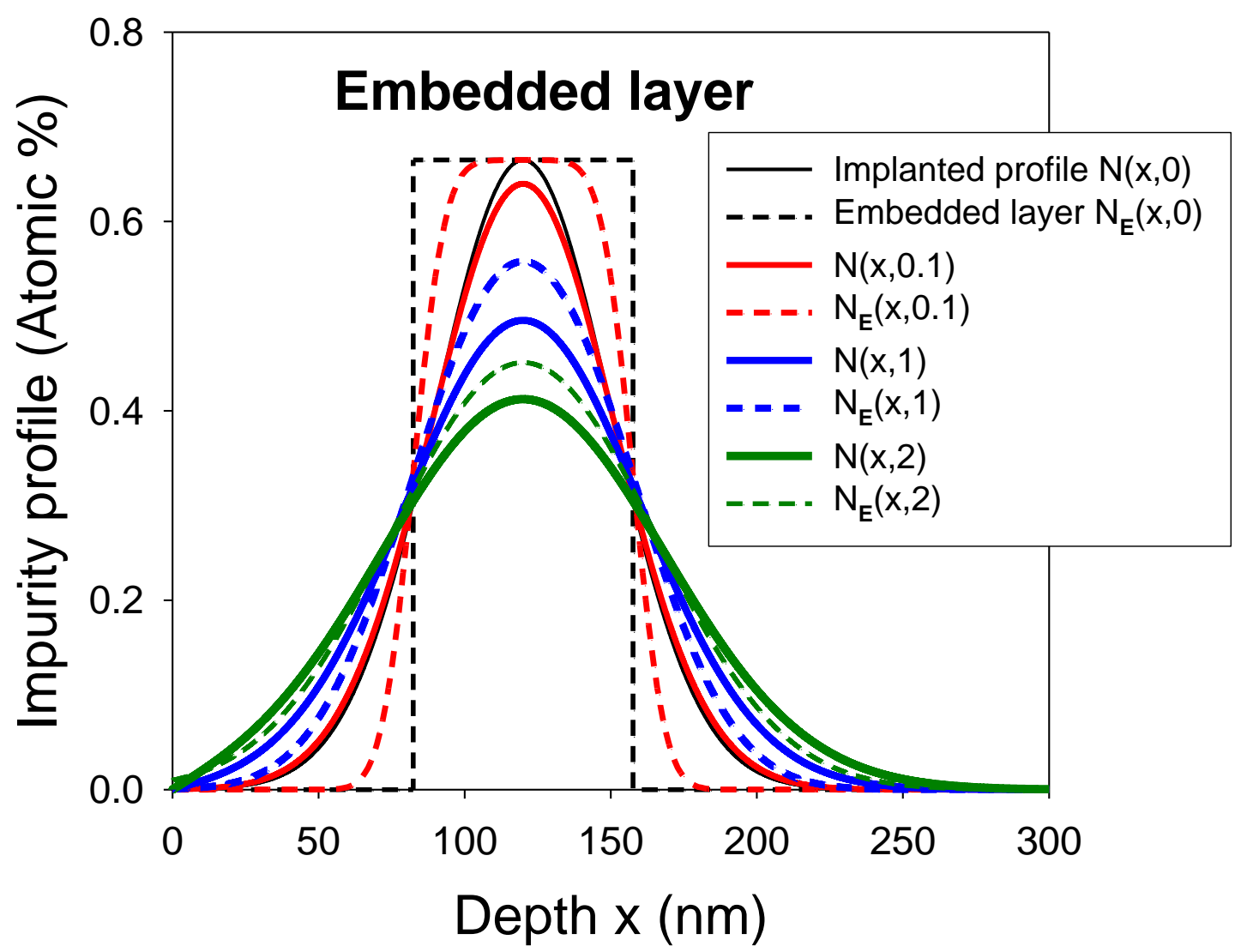

Figure 4. The diffusion of an embedded layer (broken lines), i.e. equation (14), compared to the diffusion of a Gaussian profile (solid lines) with a perfect sink at the surface, i.e. $k=1$ in (9), for different times $t$ as indicated in the figure legend. The parameters are $D=0.1 \mathrm{~nm}^{2} \mathrm{~s}^{-1}$; for the Gaussian profile (6) $R_{p}=120 \mathrm{~nm}, \Delta R_{p}=30 \mathrm{~nm}, \mathrm{~N}_{0}=A_{0}=0.66$ atomic percent.

From a comparison of the profiles in Figure 4, it can be seen that the two tails of (14) overlap with the exact diffused profile (9) for large $t$ (i.e. large $D t$ ) values. This supports the practice that only the tails of (original Gaussian) experimental diffused profile can be used for fitting to the embedded layer solution (14) to obtain a value for the diffusivity $D$. As $t$ increases $(c f . t=2)$, the whole profile can be fitted. For most experimentally determined diffusion profiles, with scatter in the measurements, the differences would be too small to make a difference.

Consider now the diffusion of a uniform layer in an semi-infinite isotropic medium, where the original profile is given by

$$
\begin{array}{ll}
N(x, 0)=N_{0} & \text { for } x<R_{p}+\Delta R_{p} \\
N(x, 0)=0 & \text { for } x>R_{p}+\Delta R_{p}
\end{array}
$$


and its solution to (1) is given in the standard references [13, 14]

$$
N_{S}(x, t)=\frac{N_{0}}{2}\left[1-\operatorname{erf}\left(\frac{x-R_{p}-\Delta R_{p}}{2 \sqrt{D t}}\right)\right]=\frac{N_{0}}{2} \operatorname{erfc}\left(\frac{x-R_{p}-\Delta R_{p}}{2 \sqrt{D t}}\right)
$$

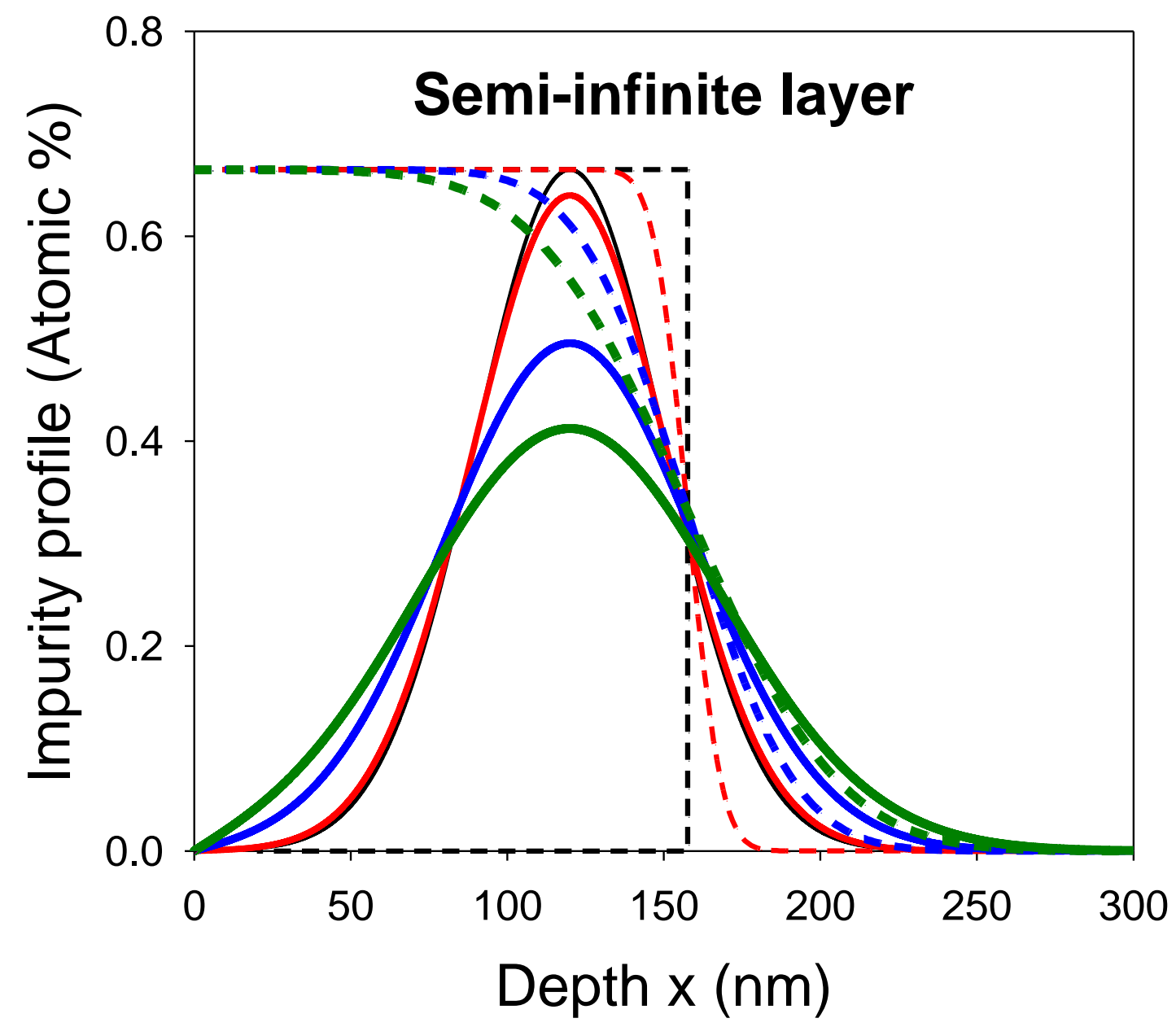

Figure 5. The diffusion of a semi-infinite layer (broken lines), i.e. equation (16), compared to the diffusion of a Gaussian profile (solid lines) with a perfect sink at the surface, i.e. $k=1$ in (9), for different times $t$ as indicated in the figure legend. The parameters are $D=0.1 \mathrm{~nm}^{2} \mathrm{~s}^{-1}$; for the Gaussian profile (6) $R_{p}=120 \mathrm{~nm}, \Delta R_{p}=30 \mathrm{~nm}, \mathrm{~N}_{0}=A_{0}=0.66$ atomic percent.

In Figure 5 this solution is compared to (9) for a perfect sink at the surface. As with the previous case, the tail of (16) overlaps with the exact diffused profile (9) for large $t$ (i.e. large $D t$ ) values. This supports the practice that only the tail of the (original Gaussian) experimental diffused profile can be used for fitting to the semi-infinite layer solution (16) to obtain a value for the diffusivity $D$. However, it is clear that only a very small portion of the profile can be fitted. This together with the difficulty 
in choosing the appropriate parameters (e.g. $N_{0}$ ) for the semi-infinite layer, makes this fitting procedure rather unreliable for obtaining good values for the diffusivity $D$.

The reason why there is agreement between these two approximate solutions and the exact diffusion profile for large $D t$ values follows from using only the first term of the asymptotic expansion of the error function, viz.. equation 7.1.23 in Abramowitz \& Stegun [15]

$$
\operatorname{erf} z \sim 1-\frac{1}{\sqrt{\pi} z} e^{-z^{2}}
$$

The proof shall only be given for the semi-infinite layer. For the embedded layer a very similar method is followed with same end result. For large argument values (16) becomes

$$
N_{S}(x, t) \sim \frac{N_{0}}{2}\left[1-\frac{2 \sqrt{D t}}{\sqrt{\pi\left(x-R_{p}-\Delta R_{p}\right)}} \exp \left[-\left(\frac{x-R_{p}-\Delta R_{p}}{2 \sqrt{D t}}\right)^{2}\right]\right]
$$

Using the similar approximations as above, for $x>R_{p}+\Delta R_{p}$, the exponential term in (18) becomes

$$
\exp \left[-\left(\frac{x-R_{p}-\Delta R_{p}}{2 \sqrt{D t}}\right)^{2}\right] \approx \exp \left(-\frac{x^{2}}{4 D t}\right)
$$

and

$$
N_{S}(x, t) \approx \frac{N_{0}}{2}\left[1-\frac{2 \sqrt{D t}}{\sqrt{\pi\left(x-R_{p}-\Delta R_{p}\right)}}\right] \exp \left(-\frac{x^{2}}{4 D t}\right)
$$

Since the exponential term dominates the pre-exponential term for large $x$ values (i.e. $x>>1)$

$$
N_{S}(x, t) \sim \exp \left(-\frac{x^{2}}{4 D t}\right)
$$

In the previous section it was shown that for large $x$ values, the exact solution (9) can be approximated by (12). For $x \gg R_{p}$ and $4 D t>>2 \Delta R_{p}$ and using the binomial expansion, (12) becomes 


$$
\frac{A_{0} \cdot \Delta R_{p}}{\sqrt{2 D t+\Delta R_{p}^{2}}} e^{\left(-\frac{\left(x-R_{p}\right)^{2}}{4 D t+2 \Delta R_{p}^{2}}\right)} \approx \frac{A_{0} \cdot \Delta R_{p}}{\sqrt{2 D t+\Delta R_{p}^{2}}} e^{\left(-\frac{x^{2}}{4 D t}\right)} \sim \exp \left(-\frac{x^{2}}{4 D t}\right)
$$

There is another equation often used to extract the diffusivity $D$ from experimental data. Myers et al. [5] incorrect derived a solution to the Fick diffusion differential equation for the following (initial) Gaussian profile

$$
N(x, 0)=\frac{K}{\sqrt{\pi D t_{0}}} \exp \left(-\frac{x^{2}}{4 D t_{0}}\right)
$$

where $K$ and $t_{0}$ are adjustable parameters. Note that this equation is slightly different to the normal Gaussian equation in lacking a factor $1 / 2$ in the pre-exponential constant term. For their solution, Myers et al. used the boundary condition for a perfectly reflecting boundary. The incorrect solution is then given by

$$
N_{M}(x, t)=\frac{K}{\sqrt{\pi D\left(t+t_{0}\right)}} \exp \left(-\frac{x^{2}}{4 D\left(t+t_{0}\right)}\right)
$$

This diffused profile is still Gaussian but broader than the original. A simple linear fit to the square of the widths of the profiles yields the diffusion coefficient $D$. The width $W(t)$ of the diffused profile is defined by

$$
W(t)=\frac{1}{2} N_{M}(0, t)
$$

From (24) follows the relation

$$
W^{2}(t)=4 D t \ln (2)+W^{2}(0)
$$

The slope of $W^{2}(t)$ against $t$ yields $D$. 
The latter equation has been used very successfully in numerous publications to obtain the diffusion coefficients of implanted materials [5, 9, 10, 1822 - 25].

It is interesting to compare dominating term (12) of the exact solution (9) to the Myers solution (24) when one makes the translation $x \rightarrow x-R_{p}$, i.e. to the equation

$$
N_{M}(x, t)=\frac{K}{\sqrt{\pi\left(D t+\Delta R_{P} / 2\right)}} \exp \left(-\frac{\left(x-R_{p}\right)^{2}}{\left.4 D t+2 \Delta R_{p}{ }^{2}\right)}\right)
$$

where we have used the relationship $2 D t_{0}=\Delta R_{p}^{2}$. By choosing an appropriate value for $K$ (or $A_{0}$ ) it is clear that the two expressions in (12) and (27) are equal to each other. This is the reason why the Myers et al. [5] equations (24) - (26) have been so successfully applied in the past to determine the diffusion coefficient of implanted species.

\section{SUMMARY}

For many practical reasons, the diffusion of an implanted element in a substrate often needs to be investigated. The implanted profile in an isotropic substrate of a monoenergetic ion species is usually very near a Gaussian profile. None of the standard textbooks give a solution to the Fick diffusion differential equations for an initially Gaussian profile.

In this paper an exact solution of an initially Gaussian profile is presented. In general, most solutions to the Fick diffusion equation are usually limited to two cases viz. that of a perfect sink at the surface and that of a perfectly reflecting surface plane at the surface origin. The solution presented here, is a general one, also covering the diffusion within the abovementioned two limiting cases. An analysis of the solutions for these two limiting cases shows that at low diffusion times the main effect is a nearly symmetric broadening of the implanted profile. At the origin and for longer diffusion times the profile deviates significantly from Gaussian. Naturally, this exact solution does not hold when there is an additional driving force such as surface segregation $[17,19,20]$. 
In the literature often only approximate solutions to the Fick diffusion differential equation are used in diffusion studies of implanted materials. A short review was given of such approximate equations and methods. This analysis also shows why the approximate (incorrect) equation by Myers et al. [5] has been so successfully employed in the past; also by our group.

\section{REFERENCES}

1. L. Ruben, J. Poate, The Industrial Physicist 9 (2003) 12.

2. IAEA-TECDOC-1607 (2008) Ion beam applications in bulk modifications of insulators.

3. IAEA-TECDOC-1165 (2000) Surface modification of materials by ion implantations for industrial and medical applications.

4. G. Dearnaley, Thin Solid Films 107 (1983) 315.

5. S.M. Myers, S.T. Picraux, T.S. Prevender, Phys. Rev. B 9 (1974) 3953.

6. S.M. Myers, J. Vac. Sci. Technol. 15 (1978) 1650.

7. J.J. Hamilton, N.E.B. Cowern, J.A. Sharp, K.J. Kirkby, E.J.H. Collart, B. Colombeau, M. Bersani, D. Giubertoni, A. Parisini, Appl. Phys. Lett. 89 (2006) 042111.

8. M. Uematsu, J. Appl. Phys. 11 (2012) 073517.

9. E. Friedland, N.G. van der Berg, T.T. Hlatshwayo, R.J., Kuhudzai, J.B. Malherbe, E. Wendler, W. Wesch, Nucl. Instrum. Methods Phys. Res. B 286 (2012) 102.

10. T.T. Hlatshwayo, J.B. Malherbe, N.G. van der Berg, L.C. Prinsloo, A.J. Botha, E. Wendler, W. Wesch, Nucl. Instrum. Methods Phys. Res. B 274 (2012) 120.

11. A. Kozakiewicz, B. Ghosh, P. Chakraborty, T. Derry, S. R. Naidoo, P. Franklyn, IEEE Photonics J. 4 (2012) 205. 
12. M. Salamony, A. Strohm, T.Voss, P. Laitinen, I. Riihimäki, S. Divinskiy, W. Frank, J. Räisänen, H. Mehrer, Phil. Mag. 84 (2004) 737.

13. J. Crank, “The Mathematics of Diffusion”, Clarendon, Oxford, 1975.

14. B.I. Boltaks, Diffusion in Semiconductors, Infosearch Ltd, London, (1963) Chap. 4.

15. M. Abramowitz and I.A. Stegun, Handbook of Mathematical Functions, National Bureau of Standards, Washington, 1964, Chapter 5.

16. A. Strohm, PhD Thesis (2002), Stuttgart University, Stuttgart, Germany.

17. J.B. Malherbe, Crit. Rev. Solid State Mater. Sci. 19 (1994) 128.

18. E. Friedland, T. Hlatshwayo, N van der Berg, Phys Status Solidi C 10 (2013) 208.

19. J.B. Malherbe, Crit. Rev. Solid State Mater. Sci. 19 (1994) 55.

20. O.S. Odutemowo, J.B. Malherbe, L. Prinsloo, D.F. Langa and E Wendler, Nucl. Instrum. Methods Phys. Res. B 371 (2016) 332.

21. N. Moncoffre, G. Barbier, E. Leblond, Ph. Martin, H. Jaffrezic, Nucl. Instrum. Methods Phys. Res. B 140 (1998) 402.

22. J. Philibert, Diffusion et Transport de Matière dans les Solides, Les Ed. de Physique, 1985.

23. E. Friedland, N.G. van der Berg, J.B. Malherbe, J.J. Hancke, J.R.N. Barry, E. Wendler, W. Wesch, J. Nucl. Mater. 410 (2011) 24.

24. T.T. Hlatshwayo, J.B. Malherbe, N.G. van der Berg, A.J. Botha, P. Chakraborty, Nucl. Instrum. Methods Phys. Res. B 273 (2012) 61.

25. D.F.Langa, N.G. Van der Berg, E. Friedland, J.B. Malherbe, A.J. Botha, P. Chakraborty, E. Wendler, W. Wesch, Nucl. Instrum. Methods Phys. Res. B $273(2012) 68$ 